\title{
PARTITIONING CONTINUOUS CURVES
}

\author{
R. H. BING
}

\section{INTRODUCTION}

1. The continuous curve. By a continuous curve we mean a compact, locally connected, metric continuum. For definitions of locally connected, continuum, and other terms used in this paper, see either [18] or [21]. Those who like to visualize topology concretely may wish to think of a continuous curve as a chunk out of Euclidean 3 -space- one that is connected (all in one piece), one that is bounded (lies on the interior of a sphere), and one that is locally connected (nearby points belong to small connected subsets). A wad of paper, an irregularly shaped rock, or the earth itself may be considered as examples. However, our remarks about continuous curves will apply equally well to those in Euclidean spaces of all dimensions and to those in a Hilbert space.

When Jordan first introduced the term continuous curve, he defined it analytically to be the image (in the plane) of a straight line interval under a continuous transformation. It was not until over twenty years later that it was discovered that any compact locally connected metric continuum was the image of a straight line interval under a continuous transformation and conversely. This interesting and unusual discovery adds spice to the study of mathematics [24, p. 12]. Another interesting aspect of this discovery is that it was made independently by two mathematicians, Hahn and Mazurkiewicz. Since Peano had shown earlier that a square plus its interior is the image of a straight line interval, a continuous curve is sometimes called a Peano continuum.

In this discussion we shall be interested in the continuous curve itself and not the continuous transformation of an interval. Hence, we use the definition in the first paragraph rather than the analytic one. In this discussion we shall be interested in the structure of a continuous curve.

2. Examples of continuous curves. A straight line interval, a square plus its interior in the plane, and a cube plus its interior in 3 -space are examples of continuous curves. In fact, any closed $n$-cell or $n$-simplex is an example. One can get a less familiar continuous

An address delivered before the Minneapolis meeting of the Society, September 6, 1951, by invitation of the Committee to Select Hour Speakers for Annual and Summer Meetings; received by the editors March 5, 1952. 
curve by taking a continuum which is the sum of a finite number of dissimilar continuous curves.

If $M$ is any compact point set whatever, it is possible to obtain a continuous curve by adding to $M$ a countable collection of arcs. We use this fact in constructing the following two examples of continuous curves which we shall use.

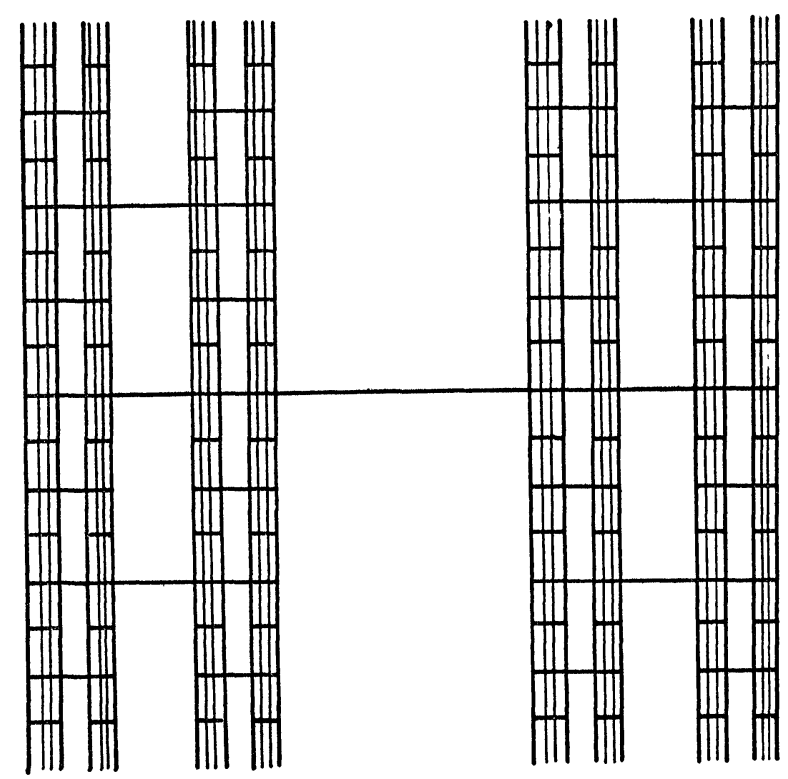

FIG. 1

Example A. Let $M$ be the cartesian product of a straight line interval and a Cantor set. Form a continuous curve by adding to $M$ a decreasing sequence of horizontal straight line intervals as indicated in Figure 1. These intervals extend from $(0,1 / 2)$ to $(1,1 / 2)$, and $(0,1 / 4)$ to $(1 / 3,1 / 4)$, from $(2 / 3,1 / 4)$ to $(1,1 / 4)$, from $(0,3 / 4)$ to $(1 / 3,3 / 4), \cdots$.

Example B. It is shown in [5] that there is a bounded continuum $S$ in Euclidean 3-space that is irreducible with respect to separating 3 -space and has the property that if two of its subcontinua intersect, one is a subset of the other. The continuum $S$ is 2-dimensional. It is called hereditarily indecomposable because no subcontinuum of it is the sum of two proper subcontinua of the subcontinuum. A continuous curve $M$ may be formed by adding to $S$ the sum of a decreasing sequence of mutually exclusive arcs. If $K$ is a subset of $M$ irreducible 
with respect to separating $M$, each component of $K$ is indecomposable.

3. Partitioning. An interval can be partitioned. This fact is used in defining the integral of a continuous function over an interval. $\left(\int_{a}^{b} f(x) d x=\lim \sum f\left(\xi_{i}\right) \Delta x_{i}\right.$ where $\xi_{i}$ is a point of and $\Delta x_{i}$ is the length of the $i$ th element of the partitioning of $[a, b]$.)

In studying a square plus its interior it is sometimes convenient to partition it into small pieces. For example, in integrating over a square region, we may partition it into small rectangles (if we use rectangular coordinates) or into pieces of other shapes (in polar coordinates for instance).

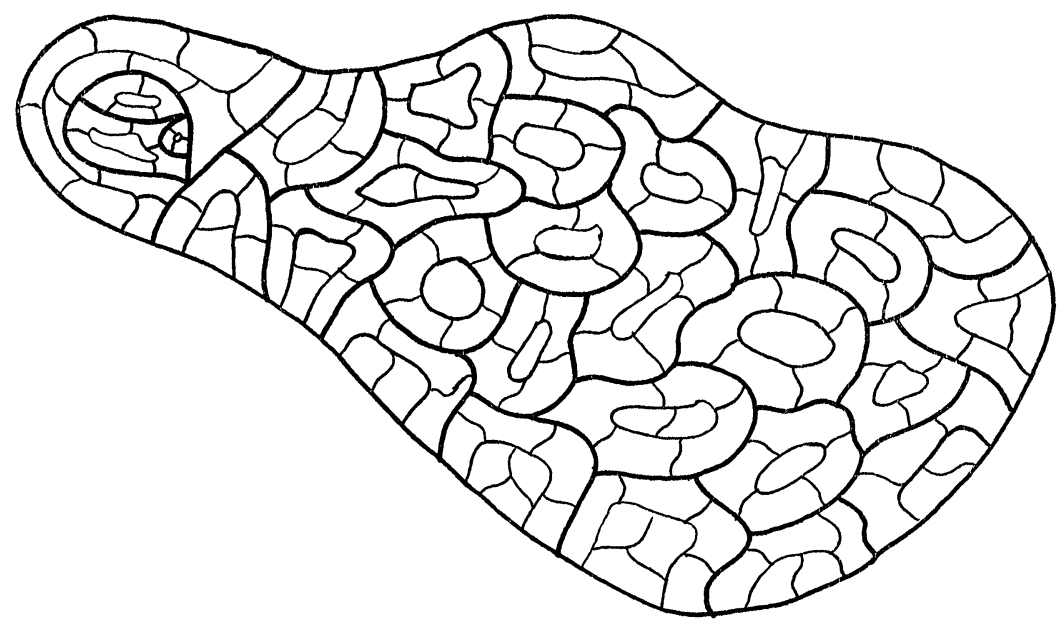

FIG. 2

A partitioning of a continuous curve $M$ is a finite collection $G$ of mutually exclusive connected open subsets of $M$ whose sum is dense in $M$ [6]. If the mesh of $G$ is less than $\epsilon$ (each element of $G$ is of diameter less than $\epsilon), G$ is called an $\epsilon$-partitioning. The essential difference between a partitioning and a grating $[13, p .279]$ is that the elements of a partitioning are connected. It is much like a grille decomposition [15].

In defining the integral over a plane domain $R\left(\iint_{R} f(x, y) d S\right.$ $=\lim$ (mesh of partitioning $\rightarrow 0) \sum f\left(\xi_{i}, \eta_{i}\right) \Delta S_{i}$ ) we do not need to impose the conditions that the elements of the partitioning are either connected, mutually exclusive, or open. However, we shall be using partitionings in connections where these properties are important. 
4. Decreasing sequence of partitionings. If $G$ and $H$ are two partitionings of the same continuous curve, $G$ is called a refinement of $H$ if each element of $G$ is a subset of an element of $H$. In Figure 2, the partitioning represented by light lines is a refinement of the one represented by heavy lines. A sequence $G_{1}, G_{2}, \cdots$ of partitionings is called a decreasing sequence if, for each positive integer $i, G_{i+1}$ is a refinement of $G_{i}$ and the mesh of $G_{i}$ approaches 0 as $i$ increases without limit.

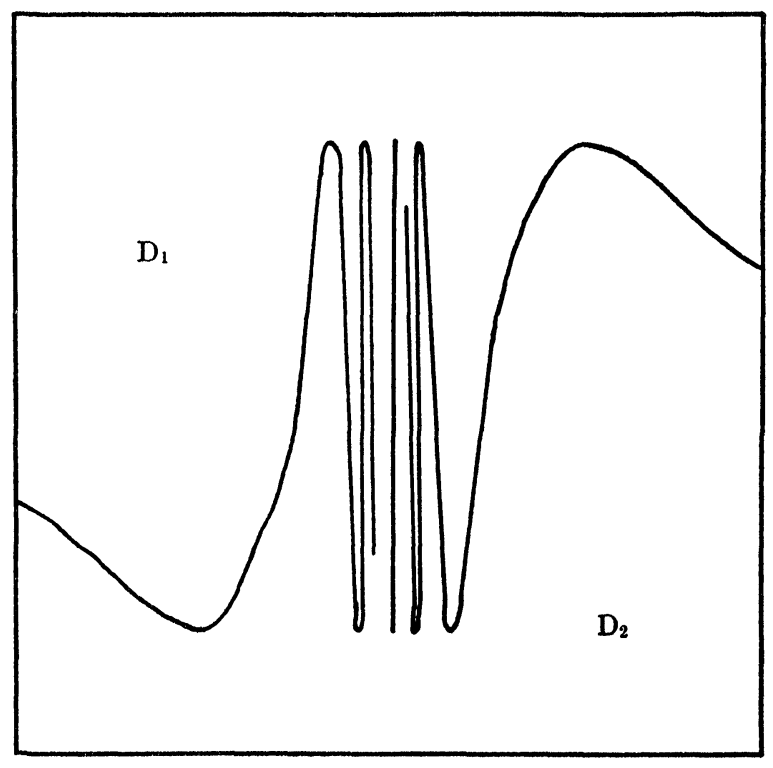

FIG. 3

While it is apparent that there is a decreasing sequence of partitionings of a square plus its interior, it is not so obvious that every continuous curve has such a sequence of partitionings. However, it is true that any continuous curve has such a sequence. This has given us new information about the continuous curve.

5. Purpose of paper. It is only within the last three years that it has been known that each continuous curve can be partitioned $[6 ; 4$; $15 ; 16]$. It is the purpose of this paper to point out some recent developments, to show ways in which a continuous curve can be partitioned, and to point out analogies between the ways in which a square plus its interior can be partitioned and the ways in which an arbitrary continuous curve can be partitioned. Details of some proofs 
are included for completeness, but some readers may prefer to skip them.

\section{BREAKING A CONTINUOUS CURVE into two PIECES}

1. The type of division needed. If one pursues the proper attack, it is not difficult to show that any continuous curve can be partitioned. However, if one gets off on the wrong track, considerable difficulty may be encountered.

Suppose it is desired to partition a continuous curve into pieces of diameters less than a fixed positive number $\epsilon$. A straightforward method of attack is to divide the continuous curve into two pieces, divide each of the two pieces, and continue this procedure until an $\epsilon$-partitioning results. However, unless certain precautions are taken, this method does not succeed.

If $\epsilon=1, M$ is a square plus its interior, and at the first stage it is partitioned into two pieces as shown in Figure 3, the above procedure will not lead to a partitioning. The common boundary of $D_{1}$ and $D_{2}$ is a part of the closure of the graph of $y=\sin 1 / x$ and neither $D_{1}$ nor $D_{2}$ is the sum of a finite number of connected subsets each of diameter less than 1.

A set has property $S$ if, for each positive number $\epsilon$, the set is the sum of a finite number of connected subsets each of diameter less than $\epsilon$. This notion was used by Sierpinski and when R. L. Moore used it later, he called it property $S[19 ; 17 ; 18]$.

A precaution to take in partitioning a continuous curve is to divide it at each step so that the pieces have property $\mathrm{S}$.

We shall be interested in the following result [6].

TheоReм 1. If $H$ and $K$ are two mutually exclusive closed subsets of a continuous curve $M$, there are two mutually exclusive open subsets $D_{H}$ and $D_{K}$ of $M$ containing $H$ and $K$ respectively such that (a) each of $D_{H}$ and $D_{K}$ has property $\mathrm{S}$ and (b) $M=\bar{D}_{H}+\bar{D}_{K}$.

Before proving this theorem, we shall review the method of constructing a set with property $\mathrm{S}$.

2. Gradual growing process. This is a method used to form sets with property $\mathrm{S}$. Compare with $[22 ; 21]$.

A set $H$ is said to $\epsilon$-grow into a set $H^{\prime}$ in a set $M$ if $M$ contains $H^{\prime}$, each point of $H^{\prime}$ belongs to a connected $\epsilon$-subset (subset of diameter less than $\epsilon$ ) of $H^{\prime}$ that intersects $H$, and for some positive number $\delta$, $H^{\prime}$ contains all connected $\delta$-subsets of $M$ that intersect $H$.

Let $M$ be a set with property $\mathrm{S}, H$ be a subset of $M$, and $\epsilon$ be a positive number. Suppose one wishes to obtain a subset of $M$ with 
property $\mathrm{S}$ which contains $H$ but no point whose distance is more than $\boldsymbol{\epsilon}$ from $H$. Such a set can be obtained by the following gradual growing process. Let $\epsilon_{1}, \epsilon_{2}, \cdots$ be a sequence of positive numbers whose sum is no more than $\epsilon$. Let $H \epsilon_{1}$-grow into a set $D_{1}$ in $M, D_{1} \epsilon_{2}$-grow into a set $D_{2}$ in $M, \cdots, D_{i} \epsilon_{i+1}$-grow into a set $D_{i+1}$ in $M, \cdots$. Then the sum $D_{0}$ of $D_{1}, D_{2}, \cdots$ is an open subset of $M$, has property $\mathrm{S}$, contains $H$ but no point at a distance more than $\epsilon$ from $H$.

3. An attempt to divide. One might try to prove Theorem 1 by letting each of the sets $H$ and $K$ grow gradually in $M$ into mutually exclusive open subsets whose sum is dense in $M$. Some difficulty may be encountered in trying to keep the two subsets from intersecting and at the same time making their sum dense in $M$. This difficulty could be overcome if we could answer the following question in the affirmative.

QUestion. Does there exist a positive integer $n$ such that the following result holds for each continuous curve $M$, each positive number $\epsilon$, and each pair of mutually exclusive closed subsets $H$ and $K$ of $M$ ? If $R$ is a finite subset of $M$ such that each point of $R$ belongs to an arc in $M$ of diameter less than $\epsilon$ that intersects $H+K$, there are two collections $A_{H}$ and $A_{K}$ of arcs satisfying the following conditions:

(a) Each element of $A_{H}$ intersects $H$ but not $K$ and each element of $A_{K}$ intersects $K$ but not $H$ nor any element of $A_{H}$.

(b) Each element of $R$ belongs to an element of $A_{H}+A_{K}$.

(c) Each element of $A_{H}+A_{K}$ is of diameter less than $n \epsilon$.

We cannot let $n=1$ and always get such collections $A_{H}$ and $A_{K}$, for let $M$ be the sum of the interval from $(-10,-1)$ to $(10,1)$ and the interval from $(-10,1)$ to $(10,-1), H=\{(-10,1)\}, K$ $=\{(10,1)\}, R=\{(-10,-1),(10,-1)\}$, and $\epsilon=11$. Had the answer been in the affirmative for $n=1$, the attempts made in [15] to partition a continuous curve might have succeeded [16].

Since we do not know the answer to the above question, we shall get some other results before proving Theorem 1.

4. A crude division. Before proving Theorem 1, it is convenient to prove a weaker theorem.

TheOREM 1'. Theorem 1 is true if condition (a) is replaced by the weaker conditions that $D_{H}$ has property $\mathrm{S}$ and $D_{K}$ has only a finite number of components.

There are two essentially different methods of proving Theorem $1^{\prime}$. One method is due to Whyburn [22, Corollary, p. 137]. He considers an uncountable family of sets with property $S$ such that of any two 
of the sets, one contains the closure of the other. He shows that one of the sets can be modified so that it still has property $S$ and the complement of its closure has a finite number of components.

An essentially different method is to let $H$ grow gradually and $K$ in a wild fashion to form sets $D_{H}$ and $D_{K}$ satisfying the conditions of Theorem 1' [6]. This method is similar to another attack we shall use later so we include the details.

Details of second method of proof of Theorem 1'. The open subsets $D_{H}$ and $D_{K}$ are formed by permitting $H$ and $K$ to grow so that $H$ grows in a convergent fashion. The procedure is as follows.

We use $\rho(p, q)$ to denote the distance between $p$ and $q$.

Suppose that, at the $i$ th stage, $H$ and $K$ have been enlarged to sets $H_{i}$ and $K_{i}$ such that $\rho\left(H_{i}, K_{i}\right)>0$ and each point of $M$ belongs to an arc in $M$ of diameter less than $1 / 2^{i}$ that intersects $H_{i}+K_{i}$. Let $R_{i}$ be a finite subset of $M$ such that each point of $M$ belongs to an arc in $M$ of diameter less than $1 / 2^{i+1}$ that intersects $R_{i}$. For each point $p$ of $R_{i}$ let $A_{p}$ be an arc in $M-\bar{H}_{i}$ from $p$ to a point of $K_{i}$ if there is one; if there is no such arc $A_{p}$, let $B_{p}$ be an arc of diameter less than $1 / 2^{i}$ in $M-\bar{K}_{i}$ from $p$ to a point of $H_{i}$.

Use $H_{i}^{\prime}$ to denote $H_{i}$ plus the sum of the $B_{p}$ 's, $K_{i}^{\prime}$ to denote $K_{i}$ plus the sum of the $A_{p}^{\prime}$ 's, and $\delta_{i}$ to denote $\rho\left(H_{i}^{\prime}, K_{i}^{\prime}\right)$. Let $H_{i+1}$ $=\left\{p \mid p\right.$ lies on an arc of diameter less than $\delta_{i} / 3$ in $M$ that intersects $\left.H_{i}^{\prime}\right\}$ and $K_{i+1}=\left\{p \mid p\right.$ lies on an arc of diameter less than $\delta_{i} / 3$ in $M$ that intersects $\left.K_{i}^{\prime}\right\}$.

Since $H_{i}$ has $\epsilon_{i}$-grown into $H_{i+1}$ for $\epsilon_{i}=7 /\left(2^{i+1} \cdot 3\right)$ and the $\epsilon_{i}$ 's have a finite sum, $\sum H_{i}=D_{H}$ has property $\mathrm{S}$.

Since $K_{i}$ has only a finite number of components and each component of $K_{i+1}$ intersects $K_{i}, \sum K_{i}=D_{K}$ has only a finite number of components.

Since no point of $M$ is at a distance of more than $1 / 2^{i}$ from $H_{i}+K_{i}$, $M=\bar{D}_{H}+\bar{D}_{K}$.

5. Proof of Theorem 1. By using Theorem $1^{\prime}$, it is shown in Theorem $3^{\prime}$ of the next section that for each positive number $\epsilon$, there is an $\epsilon$-partitioning of $M$. We use this result in establishing Theorem 1. For each integer $i$, let $G_{i}$ be a $1 / 2^{i}$-partitioning of $M$.

The general idea is to let both $H$ and $K$ grow gradually into sets $D_{H}$ and $D_{K}$. Suppose at the $i$ th stage $H$ and $K$ have been enlarged to $H_{i}$ and $K_{i}$ so that $\rho\left(H_{i}, K_{i}\right)>0$ and $H_{i}+K_{i}$ intersects each element of $G_{i}$. Let $\left\{p_{j}\right\}$ be a finite number of points such that each element of $G_{i+1}$ contains an element of $\left\{p_{j}\right\}$ and each point of $\left\{p_{j}\right\}$ lies in an element of $G_{i}$.

If there is an arc in both $M-\bar{H}_{i}$ and an element of $G_{i}$ from $p_{j}$ to a 
point of $K_{i}$, let $A_{j}$ be such an arc; if there is no such arc, let $B_{j}$ be an arc in both $M-\bar{K}_{i}$ and an element of $G_{i}$ which is irreducible from $p_{j}$ to $\bar{H}_{i}$.

Let $\rho\left(H_{i}^{\prime}, K_{i}^{\prime}\right)=\delta_{i}$ where $H_{i}^{\prime}$ is the sum of $H_{i}$ and all the $B_{j}$ 's while $K_{i}^{\prime}$ is the sum of $K_{i}$ and all the $A_{j}$ 's.

Suppose $H_{i+1}=\left\{p \mid\right.$ there is an arc in $M$ of diameter less than $\delta_{i} / 3$ from $p$ to $\left.H_{i}^{\prime}\right\}$ and $K_{i+1}=\{p \mid$ there is an arc in $M$ of diameter less than $\delta_{i} / 3$ from $p$ to $\left.K_{i}^{\prime}\right\}$.

The sets $H_{i}$ and $K_{i}$ have $\epsilon_{i}$-grown into $H_{i+1}$ and $K_{i+1}$ where $\epsilon_{i}=7 /\left(2^{i+1} \cdot 3\right)$. Hence, the sets $D_{H}=\sum H_{i}$ and $D_{K}=\sum K_{i}$ have property S. Since $H_{i}+K_{i}$ intersects each element of $G_{i}, M=\bar{D}_{H}+\bar{D}_{K}$.

\section{Partitioning}

1. Treating S-sets as continuous curves. It is necessary that a set $M$ have property $\mathrm{S}$ in order to be partitionable. (A set $M$ is partitionable if for each positive number $\epsilon$, there is an $\epsilon$-partitioning of it.) The converse is true but it is not so obvious. However this converse follows from the facts that a continuous curve is partitionable and that any connected set with property $S$ can be imbedded in a continuous curve so that any partitioning of the continuous curve produces a partitioning of it with no larger mesh.

If $G$ is a partitioning of a continuous curve, the pieces of $G$ are not continuous curves. However, if these pieces have property $S$, they can be satisfactorily dealt with.

Suppose $M$ is a connected set with property $\mathrm{S}$ and $R(p, q)$ is the relative distance for $M$, that is, $R(p, q)$ is the greatest lower bound of the diameters of all connected subsets of $M$ containing $p+q$. It may be shown that $R(p, q)$ preserves the topology of $M$ and the diameter of any connected set is not increased under $R(p, q)$. Hence, any $\epsilon$-partitioning of $M$ under the metric $R(p, q)$ is an $\epsilon$-partitioning of it under its original metric.

If we add only enough points to $M$ to make it complete under $R(p, q)$, the resulting set $M^{\prime}$ is a continuous curve. Any $\epsilon$-partitioning of $M^{\prime}$ induces an $\epsilon$-partitioning of $M$. In fact we have the following result $[6$, Lemma $1 ; 21$, pp. 154-158].

THEOREM 2. If $M$ is a connected set with property $\mathrm{S}$ with a relative distance metric, $M^{\prime}$ is the complete enclosure of $M$, and $D$ is a connected open subset of $M^{\prime}$, then $M \cdot D$ is a connected open subset of $M$.

2. Crude partitioning. Before showing that a continuous curve can be partitioned in various fashions, we show that it can be partitioned in some manner. 
TheOREM $3^{\prime}$. For each positive number $\epsilon$ and each continuous curve $M$, there is an $\epsilon$-partitioning $G$ of $M$.

We use the following corollary which results from applying Theorems $1^{\prime}$ and 2 to each component of $M$.

Corollary of Theorem 1'. Suppose that the set $M$ has property S and that $H$ and $K$ are two of its subsets such that $\rho(H, K)>0$. Then $M$ contains two mutually exclusive open subsets $D_{H}$ and $D_{K}$ containing $H$ and $K$ respectively such that $D_{H}+D_{K}$ is dense in $M, D_{H}$ has property $S$, and $D_{K}$ has only a finite number of components.

The general idea in proving Theorem $3^{\prime}$ is to use Theorem $1^{\prime}$ and divide $M$ into two pieces $D_{H}$ and $D_{K}$ so that $D_{H}$ has property $\mathrm{S}$, $D_{K}$ has only a finite number of components and is of diameter less than $\epsilon$. We apply the corollary of Theorem $1^{\prime}$ to $D_{H}$ and divide it into two pieces one of which has property $S$ and the other of which has only a finite number of components each of diameter less than $\epsilon$. The procedure is continued so as to get an $\epsilon$-partitioning of $M$.

Details of Proof of Theorem $3{ }^{\prime}$. Let $\left(p_{1}, p_{2}, \cdots, p_{n}\right)$ be a finite subset of $M$ such that no point of $M$ is at a distance as much as $\epsilon / 4$ from $\left\{p_{i}\right\}$. Let $K_{i}$ be the set of all points of $M$ that are at a distance from $p_{i}$ of less than $\epsilon / 4$ and $H_{i}$ be the set of those that are at a distance from $p_{i}$ of more than $\epsilon / 3$.

Applying Theorem $1^{\prime}$ to $M$, we find that there are mutually exclusive subsets $D_{H_{1}}$ and $D_{K_{1}}$ of $M$ containing $H_{1}$ and $K_{1}$ respectively such that $\bar{D}_{H_{1}}+\bar{D}_{K_{1}}=M, D_{H_{1}}$ has property $\mathrm{S}$, and $D_{K_{1}}$ has only a finite number of components. The components of $D_{K_{1}}$ are of diameters less than $\epsilon$ and are elements of $G$. We divide the components of $D_{H_{1}}$ further.

If $D_{H_{1}}$ intersects both $H_{2}$ and $K_{2}$, we apply the corollary of Theorem $1^{\prime}$ to $D_{H_{1}}$ and find that there are open subsets $D_{H_{2}}$ and $D_{K_{2}}$ of $D_{H_{1}}$ containing $H_{2} \cdot D_{H_{1}}$ and $K_{2} \cdot D_{H_{1}}$ respectively such that $D_{H_{2}}+D_{K_{2}}$ is dense in $D_{H_{1}}, D_{H_{2}}$ has property $\mathrm{S}$, and $D_{K_{2}}$ has only a finite number of components. The components of $D_{K_{2}}$ are elements of $G$. If $K_{2}$ does not intersect $D_{H_{1}}$, we do not add elements to $G$ at this stage but let $D_{H_{2}}=D_{H_{1}}$.

The process is continued. For each positive integer $i$ less than $n-1$ for which $D_{H_{i}}$ intersects both $H_{i+1}$ and $K_{i+1}$ we apply the corollary to $D_{H_{i}}$ and get mutually exclusive open subsets $D_{H_{i+1}}, D_{K_{i+1}}$ of $D_{H_{i}}$ containing $H_{i+1} \cdot D_{H_{i}}$ and $K_{i+1} \cdot D_{H_{i}}$ respectively such that $D_{H_{i+1}}$ $+D_{K_{i+1}}$ is dense in $D_{H_{i}}, D_{H_{i+1}}$ has property $\mathrm{S}$, and $D_{K_{i+1}}$ has only a finite number of components. If $D_{H_{i}}$ does not intersect $K_{i+1}$, we suppose $D_{K_{i+1}}=0$ and $D_{H i+1}=D_{H_{i}}$. 
The elements of $G$ are the components of $D_{K_{1}}, D_{K_{2}}, \cdots, D_{K_{n-1}}$, and $D_{H_{n-1}}$.

3. A more useful partitioning. If $G$ is an arbitrary partitioning of a continuous curve as described in Theorem $3^{\prime}$, it may not be the member of any decreasing sequence of partitionings because some element of it may not have property $\mathrm{S}$. However, if we use Theorem 1 instead of Theorem $1^{\prime}$, we obtain the following extension of Theorem $3^{\prime}$.

THeOREM 3. For each continuous curve $M$ and each positive number $\epsilon$ there is an $\epsilon$-partitioning $G$ of $M$ such that each element of $G$ has property $\mathrm{S}$.

A partitioning whose pieces have property $\mathrm{S}$ is called an $\mathrm{S}$-partitioning. If its mesh is less than $\epsilon$, it is an $\epsilon$-S-partitioning.

4. Decreasing sequence of partitionings. Theorem 2 may be applied to any set with property $S$ to get the following corollary of Theorem 3.

Corollary of Theorem 3. For each positive number $\epsilon$ and each set $M$ with property $\mathrm{S}$, there is an $\epsilon$-S-partitioning of $M$.

Using the corollary we find that if $G$ is an S-partitioning of a continuous curve and $\epsilon$ is a positive number, each piece of $G$ may be $\epsilon$-S-partitioned. Hence, we have the following useful result [6].

Theorem 4. Each continuous curve has a decreasing sequence of partitionings.

\section{Core Refinements}

In Figure 2, the partitioning represented by light lines is a core refinement of the one represented by heavy lines.

A partitioning is regular if each of its elements is the interior of its closure. This prevents pieces from having unnecessary boundary points.

If the partitioning $H$ is a refinement of the partitioning $G$, the elements of $H$ which have a boundary point in common with the boundary of an element of $G$ are called border elements of $H$. Other elements are called interior elements.

The partitioning $H$ is called a core refinement of the partitioning $G$ if the following conditions are satisfied:

(a) Each border element of $H$ is adjacent to an interior element.

(b) For each element $g$ of $G$, the closure of the sum of the interior elements of $H$ in $g$ is connected. 
(c) Both $G$ and $H$ are regular partitionings.

In [8] additional conditions were used in defining a core refinement for brick partitionings but we shall not need them here. We regard the interior of the closure of the sum of the interior elements of $H$ in $g$ as the core of $g$.

In some of the applications of partitionings, use is made of the following result.

THEOREM 5. For each continuous curve $M$ there is a decreasing sequence $G_{1}, G_{2}, \ldots$ of partitionings of $M$ such that $G_{i+1}$ is a core refinement of $G_{i}$.

Detalls of Proof. We prove this theorem by showing that if $G$ is a regular S-partitioning of $M$ and $\epsilon$ is a positive number, there is an $\epsilon$-S-partitioning $H$ which is a core refinement of $G$.

For each element $g$ of $G$, let $H^{\prime}$ be a regular $\epsilon$-S-partitioning of $g$. There is a dendron $T$ in $g$ that intersects each element of $H^{\prime}$. Let $H^{\prime \prime}$ be a regular S-partitioning of $g$ that refines $H^{\prime}$ and has a mesh less than the distance between $T$ and the boundary of $g$.

Each element $h^{\prime \prime}$ of $H^{\prime \prime}$ with a boundary point on the boundary of $g$ is a subset of a border element of $H$. If $h^{\prime}$ is the element of $H^{\prime}$ containing $h^{\prime \prime}$, the border element $h$ of $H$ containing $h^{\prime \prime}$ is the interior of the closure of the sum of all elements $k$ of $H^{\prime \prime}$ such that there is an arc in $h^{\prime}$ from $h^{\prime \prime}$ to $k$ that does not intersect the closure of any element of $H^{\prime \prime}$ whose closure intersects $T$. All elements of $H^{\prime \prime}$ not in such a border element of $H$ are interior elements of $H$.

\section{THE CONVEXIFICATION PROBLEM}

1. The method of solution. A metric is a convex metric for a continuous curve $M$ if for each pair of points of $M$ there is a point of $M$ halfway between them. In 1928 Menger raised [14] the question as to whether or not each continuous curve has a convex metric that preserves its topology. During the following twenty years a number of contributions $[1 ; 3 ; 9 ; 10 ; 12]$ were made toward the solution of this question but it was not until the notion of partitioning was introduced that the final answer was given $[6 ; 15]$.

TheOREM 6. Each continuous curve has a convex metric.

The fact that each continuous curve has a decreasing sequence $G_{1}, G_{2}, \cdots$ of partitionings is used in showing that each continuous curve has a convex metric. Each piece of each $G_{i}$ is assigned a size and the distance between two points $p, q$ is defined in terms of the sizes of the chains from $p$ to $q$ whose links are elements of the $G_{i}$ 's. 
2. Definition of convex metric. Let $G_{1}, G_{2}, \ldots$ be a sequence of partitionings of $M$ such that $G_{i+1}$ is a core refinement of $G_{i}$ and the mesh of $G_{i+1}$ is less than one third the distance between any two nonadjacent elements of $G_{i}$.

The elements of $G_{1}$ are assigned a size of $1 / 2$. Each border element of $G_{i+1}$ is assigned a size equal to one half the size of the element of $G_{i}$ containing it. Each interior element of $G_{i+1}$ is given a size equal to $1 /\left(2^{i+1} \cdot N_{i+1}\right)$ where $N_{i+1}$ is the number of elements in $G_{i+1}$.

If $K$ is a continuum which is equal to the closure of the sum of the elements of $G_{i}$, the $i$ th size of $K$ is the sum of the sizes of the elements of $G_{i}$ in $K$. We define $E_{i}(p, q)$ as the minimum of the $i$ th sizes of all continua containing $p+q$. It may be noted that if $E_{i}(p, q)$ is the $i$ th size of $K$, then $K$ is the sum of a chain $C_{1}, C_{2}, \cdots, C_{n}$ of continua such that $C_{1}$ contains $p, C_{n}$ contains $q, C_{j}$ intersects $C_{k}$ if and only if $|j-k| \leqq 1$ and $C_{j}$ is the closure of an element of $G_{i}$. The convex metric for $M$ is $D(p, q)=\lim E_{i}(p, q)$.

We note that $D(p, p)=0$ and $D(p, q) \geqq 0$. Also $D(p, q)=D(q, p)$ because $E_{i}(p, q)=E_{i}(q, p)$.

If $p, q, r$ are three points, $E_{i}(p, q)+E_{i}(q, r) \geqq E_{i}(p, r)$ because if $K_{p q}$ and $K_{q r}$ are continua with $i$ th sizes containing $p+q$ and $q+r$ respectively, the $i$ th size of the continuum $K_{p q}+K_{q r}$ is no more than the sum of the $i$ th sizes of $K_{p q}$ and $K_{q r}$. Hence, $D(x, y)$ satisfies the triangle condition.

We now show that if $p$ is a limit point of the set $A$, then $D(p, A)=0$. Since $E_{i}(p, q)+1 / 2^{i+1} \geqq E_{i+1}(p, q), D(p, q) \leqq E_{i}(p, q)+1 / 2^{i}$. However, there is a point $q$ of $A$ such that $p+q$ belongs to the closure of the same element of $G_{i}$. Then $D(p, q) \leqq 1 / 2^{i-1}$ and $D(p, a)$ (a element of $A$ ) is not bounded from 0 .

We now show that if $p$ is not a point of the closed set $A, D(p, A)$ $>0$. Suppose $i$ is an integer such that no element of $G_{i}$ is adjacent to both an element of $G_{i}$ whose closure contains $p$ and also to an element of $G_{i}$ whose closure intersects $A$. If $\epsilon$ is the least of the sizes of the elements of $G_{i}$, we shall show that $D(p, A) \geqq \epsilon$.

If $K$ is a continuum containing $p$, intersecting $A$, and having an $(i+k)$ th size, $K$ contains $2^{k}$ elements $g$ of $G_{i+k}$ such that $g$ is a border element of $G_{i+k}$ and lies in border elements of $G_{i+1}, G_{i+2}, \cdots, G_{i+k-1}$. The size of $g$ is as much as $\epsilon / 2^{k}$ and the $(i+k)$ th size of $K$ is more than $\epsilon$. Hence, for each point $a$ of $A, E_{i+k}(p, a)>\epsilon$ and $D(p, a) \geqq \epsilon$.

We show that the metric $D(x, y)$ is convex by showing that if $p$ and $q$ are two points, there is a point halfway between them. Let $K$ be a continuum containing $p+q$ of $i$ th size $E_{i}(p, q)$. Then $K$ is the sum of two subcontinua $K_{p}$ and $K_{q}$ containing $p$ and $q$ respec- 
tively each of whose $i$ th sizes differ from one half the $i$ th size of $K$ by less than $1 / 2^{i}$. There is a point $r_{i}$ in $K_{p} \cdot K_{q}$ such that neither $E_{i}\left(p, r_{i}\right)$ nor $E_{i}\left(q, r_{i}\right)$ differs from $E_{i}(p, q) / 2$ by more than $1 / 2^{i}$. If $r$ is a limit point of $r_{1}, r_{2}, \cdots$, it is halfway between $p$ and $q$ under the metric $D(x, y)$.

Although a solution to the convexification problem has been given by means of partitionings, it would still be interesting to see the answer given by other methods.

3. Unique segments. If a circle is given a convex metric, for each point $p$ there is a point $q$ and two points $r_{1}, r_{2}$ such that both $r_{1}$ and $r_{2}$ are halfway between $p$ and $q$. There is not a unique segment from $p$ to $q$.

Although under a convex metric there is not a unique segment between each pair of points of a circle, there is a dense subset of a circle such that each pair of points of this dense subset is joined by a unique segment. In another paper I shall show that each continuous curve has such a convex metric. The result may be stated as follows.

THEOREm 7. Each continuous curve has a dense subset $R$ and a convex metric $D$ such that each pair of points of $R$ are the end points of a unique segment under $D$.

For the surface of a sphere, there is a convex metric and a positive number $\epsilon$ such that each pair of points whose distance apart is less than $\epsilon$ are end points of a unique segment. It would be interesting to know a topological characterization of such sets.

A closed $n$-cell in a Euclidean space has a convex metric such that if two segments intersect in more than one point, the sum of these two segments is a segment. It would be interesting to obtain a topological characterization of continuous curves that can be given such a convex metric.

Each continuous curve with a convex metric that gives unique segments can be shrunk to each of its points in a very nice fashion. One might wonder if those continuous curves with such nice convex metrics could be characterized by the fashion in which they can be shrunk to points.

\section{Mapping CONTINUOUS CURVES ONTO INTERVALS}

1. Introduction. We have seen that if $H$ and $K$ are two mutually exclusive closed subsets of a continuous curve $M, M$ can be divided into two mutually exclusive open subsets $D_{H}$ and $D_{K}$ containing $H$ and $K$ respectively such that each of the sets $D_{H}, D_{K}$ has property $S$. We shall now see how uncountably many such divisions can be made. 
We map $M$ onto a straight line interval $[0,1]$ so that $H$ goes into $0, K$ goes into 1 , and the inverse of each nondegenerate connected subset of $[0,1]$ has property $S[4]$. Then except for a countable number of points $x$ of $[0,1]$, the collection of components of $T^{-1}[0, x)$ and $T^{-1}(x, 1]$ is an S-partitioning of $M$. We use $(a, b]$ to denote the closed interval $[a, b]$ minus the point $a$.

2. Details of the transformation. To prove the theorem regarding the mapping of $M$ onto $[0,1]$ we first prove the following lemma.

Lemma For Theorem 8. Suppose $C_{1}$ and $C_{2}$ are mutually exclusive closed subsets of a continuous curve $M, M-C_{i}(i=1,2)$ has property $\mathrm{S}$, and $\epsilon$ is a positive number such that $C_{j}(j \neq i)$ can $\epsilon$-grow into $M-C_{i}$. Then for each positive number $\delta, M$ contains mutually exclusive open subsets $D_{1}$ and $D_{2}$ satisfying the following conditions:

(a) $C_{i}$ can $\delta$-grow into $D_{i}$ and $(\epsilon+\delta)$-grow into $M-D_{j}$.

(b) $M-\left(D_{1}+D_{2}\right)$ can $\delta$-grow into $M-\left(C_{1}+C_{2}\right)$.

(c) $D_{i}$ and $M-D_{i}$ have property $\mathrm{S}$.

Proof of Lemma. Let $G$ be a $\gamma$-S-partitioning of $M-\left(C_{1}+C_{2}\right)$ where $\gamma$ is less than either $\delta$ or $\rho\left(C_{1}, C_{2}\right) / 3$.

Let $A_{i}$ be a finite collection of arcs in $M-C_{j}(i \neq j)$ such that each of these arcs is of diameter less than $\epsilon$, each intersects $C_{i}$, and each element of $G$ intersects both an arc of $A_{1}$ and also an $\operatorname{arc}$ of $A_{2}$.

Let $G^{\prime}$ be a $\gamma^{\prime}$-S-partitioning of $M-\left(C_{1}+C_{2}\right)$ which refines $G$, where $\gamma^{\prime}$ is less than the distance between $C_{i}$ and any arc of $A_{j}(i \neq j)$. Let $R^{\prime}$ be the closure of the sum of all elements $g$ of $G^{\prime}$ such that $g$ either lies in an element of $G$ not adjacent to $C_{1}+C_{2}$ or $g$ lies in an element of $G$ adjacent to $C_{i}$ and $\bar{g}$ intersects an arc in $A_{i}(i \neq j)$. Then $R$ is the sum of $R^{\prime}$ and the closure of all elements $g^{\prime}$ of $G^{\prime}$ of the following sort: $g^{\prime}$ lies in an element $g$ of $G$ which is adjacent to $C_{i}$ but $R^{\prime}$ separates $g^{\prime}$ from $C_{i}$ in $g+C_{i}$.

We define $D_{i}$ to be the interior of the closure of the sum of $C_{i}$ and all elements of $G^{\prime}$ which do not lie in $R$ but lie in an element of $G$ adjacent to $C_{i}$.

Each point of $D_{i}-C_{i}$ belongs to an arc intersecting $C_{i}$ and lying in both $D_{i}$ and also in the closure of an element of $G$. This shows the first part of condition (a) is satisfied. An arc showing that the second part of this condition is satisfied may be obtained by going out an element of $A_{i}$ and then continuing in the closure of an element of $G$.

To see that condition (b) is satisfied, note that each point of $M$ $-\left(C_{1}+C_{2}\right)$ belongs to an arc that intersects $R$ and lies in both $M-\left(C_{1}+C_{2}\right)$ and also in the closure of an element of $G$.

Condition (c) is satisfied because $G^{\prime}$ is an S-partitioning. 
THEOREM 8. If $H$ and $K$ are mutually exclusive closed subsets of the continuous curve $M$, there is a continuous transformation $T$ of $M$ onto the interval $[0,1]$ such that (a) $T(H)=0$ and $T(K)=1$, (b) if $W$ is a nondegenerate connected subset of $[0,1], T^{-1}(W)$ has property $\mathrm{S},(\mathrm{d})$ if 0 belongs to $W$, each component of $T^{-1}(W)$ intersects $H$ and if 1 belongs to $W$, each component of $T^{-1}(W)$ intersects $K$.

We prove this result by repeated applications of the preceding lemma. To get a start we obtain $C_{1}=T^{-1}(0)$ and $C_{2}=T^{-1}(1)$. Let $G$ be an $\epsilon$-S-partitioning of $M$ where $\epsilon=\rho(H, K) / 3$. Then $C_{1}$ is the sum of the closures of all elements of $G$ whose closures intersect $H$ plus all points that this sum separates from $K$. Similarly, $C_{2}$ is the sum of the closures of all elements of $G$ whose closures intersect $K$ plus all points that this sum separates from $H$.

In our first application of the lemma we use $T^{-1}(0), T^{-1}(1), 1 / 2$ for $C_{1}, C_{2}, \delta$. The sets $M-\left(D_{1}+D_{2}\right), D_{1}, D_{2}$ we obtain are called $T^{-1}(1 / 2), T^{-1}[0,1 / 2), T^{-1}(1 / 2,1]$.

In our next application of the lemma we let $T^{-1}(0), T^{-1}[1 / 2,1]$ $=T^{-1}(1 / 2)+T^{-1}(1 / 2,1], 1 / 2,1 / 4$ be the $C_{1}, C_{2}, \epsilon, \delta$ of the lemma and denote by $T^{-1}(1 / 4), T^{-1}[0,1 / 4), T^{-1}(1 / 4,1]$ the sets $M$ - $\left(D_{1}+D_{2}\right), D_{1}, D_{2}$ obtained.

In general, suppose that $T^{-1}\left[0, i / 2^{i}\right]$ and $T^{-1}\left[(i+1) / 2^{i}, 1\right]$ have been defined. We apply the lemma using $T^{-1}\left[0, i / 2^{j}\right]$, $T^{-1}\left[(i+1) / 2^{i}, 1\right], 1 / 2^{j}, 1 / 2^{i+1}$ for $C_{1}, C_{2}, \epsilon, \delta$ and denote by $T^{-1}\left[(2 i+1) / 2^{i+1}\right], T^{-1}\left[0,(2 i+1) / 2^{i+1}\right), T^{-1}\left((2 i+1) / 2^{i+1}, 1\right]$ the sets $M-\left(D_{1}+D_{2}\right), D_{1}, D_{2}$ obtained.

After $T^{-1}(x)$ has been defined for all numbers of $[0,1]$ of the form $x=i / 2^{i}$, the definition of $T$ is continued in a continuous fashionthat is, $T^{-1}(x)$ is the intersection of all closed sets $T^{-1}\left[i / 2^{i},(i+1) / 2^{i}\right]$ for which $i / 2^{j} \leqq x \leqq(i+1) / 2^{i}$.

In the next section we need to use the following information about the transformation $T$.

THEOREM 9. If $T$ is a continuous transformation mentioned in Theorem 8, then except for a countable number of values $x \quad(0 \leqq x \leqq 1)$, both $T^{-1}[0, x)$ and $T^{-1}(x, 1]$ are uniformly locally connected. Furthermore, if $M$ is the closure of a uniformly locally connected domain $D$ and $B$ is a subset of the boundary of $D$, except for a countable number of values $x$, each point of $T^{-1}(x) \cdot B$ is a limit point of both $T^{-1}[0, x) \cdot B$ and $T^{-1}(x, 1] \cdot B$.

A set $D$ is uniformly locally connected if for each positive number $\epsilon$ there is a positive number $\delta$ such that each pair of points of $D$ 
whose distance apart is no more than $\delta$ belongs to a connected subset of $D$ of diameter less than $\epsilon$.

The proof of Theorem 9 is contained in the proof of Theorem 5 of [4].

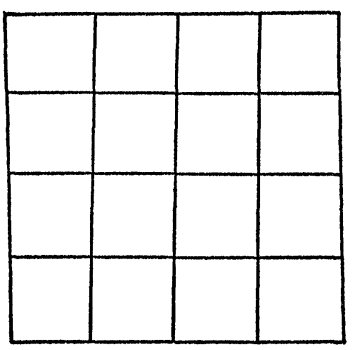

a

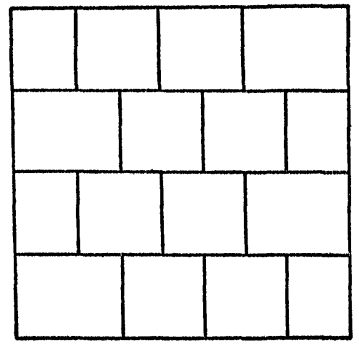

b

FIG. 4

\section{BRICK PARTITIONING}

Figure 4 shows two ways in which a square plus its interior may be partitioned. The partitioning represented in Figure $4 \mathrm{~b}$ is called a brick partitioning. A partitioning $G$ is a brick partitioning if each of its elements is uniformly locally connected and equal to the interior of its closure while the interior of the sum of the closures of each pair of elements of $G$ is uniformly locally connected. If $G$ is a brick partitioning, not only can the elements of $G$ be subdivided so as to form a partitioning $G^{\prime}$ which refines $G$ but also adjacent elements of $G$ and part of their common boundary can be added so as to form a partitioning $G^{\prime \prime}$ such that $G$ refines $G^{\prime \prime}$.

In Figure 2, the left-most piece of the partitioning shown by heavy lines is not uniformly locally connected. Also, the interior of the sum of the closures of the two left-most pieces is not uniformly locally connected. If the three left-most pieces are replaced by the interior of the sum of their closures, a brick partitioning results.

Wilder showed [24, Theorem 3.4, p. 79] that if $p$ is a point of a continuous curve $M$ and $D$ is a domain containing $p$, there is a uniformly locally connected domain $E$ containing $p$ and lying in $D$. However, to get a brick partitioning, we need to know that there is such a domain $E$ such that $M-\bar{E}$ is uniformly locally connected. We get this result from Theorems 8 and 9.

It would be interesting to know if a modification of the methods used by Wilder would give this result. It appears that Moise had this in mind in [15].

Repeated applications of Theorems 8 and 9 show the following result $[4]$. 
THEOREM 10. Each continuous curve has a decreasing sequence of brick partitionings.

The first part of Theorem 9 is used in getting the elements of the partitionings to be uniformly locally connected and the second part is used to get the interior of the sum of the closures of two elements to be uniformly locally connected.

\section{THE BOUNDARIES OF THE PIECES}

1. Locally connected boundaries. In partitioning a square plus its interior as shown in Figure 4, the boundaries of the pieces are continuous curves and can be partitioned. If $M$ is a finite complex and $G$ is the collection of simplexes of $M$ which are not part of the boundary of any other simplex, the elements of $G$ have boundaries which are the sums of a finite number of continuous curves. These boundaries can be partitioned. ( $G$ may not be a brick partitioning.)

One might wonder if each continuous curve can be partitioned so that the boundaries of the pieces are locally connected. However, Example A (Figure 1) described in $\$ I$ shows that this is not always possible. If the continuous curve is partitioned into pieces of diameters less than 1, each piece which is not a subset of a horizontal line has a boundary with uncountably many components.

However, in the partitioning of Example A, each component of the boundary of a piece of a partitioning is either a point or an interval and is therefore locally connected. One might wonder if each continuous curve can be partitioned so that the components of the boundaries of the pieces are locally connected. Example B of $\S I$ shows that this is not always possible.

It would be interesting to be able to characterize the types of continuous curves that can be partitioned into pieces with nice boundaries.

2. Dimensionally regular partitionings. Suppose that $M$ is an $n$-dimensional continuous curve. It is known that for each positive number $\epsilon$ there is an open $\epsilon$-covering $H$ of $M$ such that no point of $M$ is covered by more than $n+1$ elements of $H$ [11].

A partitioning $G$ of the $n$-dimensional continuous curve $M$ is called dimensionally regular if it is regular (each piece is the interior of its closure) and if the following inequality is satisfied when $j \leqq n+1$.

Dimension (intersection of any $j$ boundaries) $\leqq n+1-j$.

In another paper I shall prove the following result. 
THEOREM 11. There is a decreasing sequence of dimensionally regular partitionings for each n-dimensional continuous curve.

It may be noted that the boundaries of the pieces will be of dimension less than $n$.

\section{Applications of PARTItionings}

The study of partitionings throws light on the structure of continuous curves and is of interest aside from its applications. In fact, I regard the result that any continuous curve is partitionable as more interesting and topologically important than any of its applications.

The first use made of the fact that any continuous curve is partitionable was to solve the convexification problem as mentioned in $\S \mathrm{V}$.

In studying a continuous curve $M$, one may consider a decreasing sequence of finite open coverings of $M$. A decreasing sequence of partitionings might be used instead. For example, in showing that each connected open subset of a continuous curve is arcwise connected, one may use chains whose links are connected open sets to define the arcs [18]. Chains whose links are pieces of partitionings could be used instead. This was done in the following application.

1. The Kline sphere characterization. A nondegenerate continuous curve $M$ is topologically equivalent to the surface of a sphere if and only if it satisfies the following two conditions:

(a) No pair of points separates $M$.

(b) Each simple closed curve separates $M$.

Several mathematicians contributed to the proving of this result ([25] and other references in [7]). The final link was in showing that conditions (a) and (b) also imply that no arc separates $M$ [7]. This was done by showing that if there is an arc which separates $M$, there is a simple closed curve that does not. The simple closed curve was constructed by means of chains whose links are open sets. By using the notion of brick partitioning, the above result was strengthened [4] by showing that condition (b) could be replaced by the following weaker condition:

(b') Each simple closed curve whose complement has property $\mathrm{S}$ separates $M$.

2. Basis with connected intersections. A countable basis for a continuous curve is a countable collection $D_{1}, D_{2}, \cdots$ of open subsets such that if $p$ is a point and $E$ is an open set containing $p$ there is an integer $i(p, E)$ such that $D_{i}$ contains $p$ and lies in $E$. If $S$ is a square plus its interior, there is a countable basis for $S$ whose elements are 
the intersections of $S$ with interiors of circles with rational radii and rational centers. The intersection of any two elements of such a basis is connected. One might wonder if each continuous curve has such a basis.

E. E. Floyd noticed that if $G$ is a brick partitioning of $M$ and $H$ is the collection of all open sets $h$ such that for some point $p, h$ is the interior of the sum of the closures of all elements of $G$ whose closures contain $p$, then the intersection of any two elements of $H$ is connected. Consequently, Floyd and I showed [8] that any continuous curve has a countable basis such that the intersections of the elements of this basis are connected and uniformly locally connected.

3. Characterizing 3-space. Suppose $H$ and $K$ are two sets such that for each positive number $\epsilon$ there are $\epsilon$-partitionings $G_{H}$ and $G_{K}$ of $H$ and $K$ respectively and a 1-1 correspondence between the elements of $G_{H}$ and the elements of $G_{K}$ such that two elements of $G_{H}$ have a common boundary point if and only if the corresponding elements of $G_{K}$ have a common boundary point. One might wonder if this implies that $H$ is homeomorphic to $K$. It does not because if $H$ is a square plus its interior and $K$ is the sum of $H$ and a straight line interval that intersects $H$ in just one point, there are such partitionings $G_{H}$ and $G_{K}$. However, it is possible to use the notion of partitionings to characterize certain sets.

The two-manifold can be partitioned so that the boundary of the elements of the partitionings are simple closed curves. Some 3-manifolds can be partitioned so that the boundaries of the pieces are simple surfaces (topologically equivalent to the surface of a sphere). It is an open question as to whether or not all compact 3-manifolds without boundary have this property. The 3-manifold obtained by adding an ideal point at infinity to ordinary Euclidean 3 -space is called a 3-sphere. In fact any set homeomorphic to it is called a 3-spherefor example, the surface of a sphere in Euclidean 4-space. Using the notion of partitioning, we can get the following characterization of a 3-sphere [2].

$A$ necessary and sufficient condition that a continuous curve $M$ be a 3-sphere is that one of its decreasing sequences of regular partitionings $G_{1}, G_{2}, \cdots$ have the following properties:

(a) The boundary of each element of $G_{i}$ is a simple surface.

(b) If the boundaries of two elements of $\sum G_{i}$ intersect, this intersection is a 2-cell.

(c) The intersection of the boundaries of three elements of $G_{i}$ is onedimensional at each of its points. 
(d) If $g$ is an element of $G_{i-1}(g=M$ if $i=1)$ the elements of $G_{i}$ may be ordered $g_{1}, g_{2}, \cdots, g_{n}$ so that the boundary of $g_{j}(j=1,2, \cdots, n)$ intersects the boundary of $g$ plus the boundary of $g_{1}+g_{2}+\cdots+g_{j-1}$ in a connected set.

4. Simultaneous partitionings. In a forthcoming paper [20] Garth Thomas shows that if the continuous curve $M_{1}$ is imbedded in the continuous curve $M_{2}$, there is a partitioning $G_{2}$ of $M_{2}$ such that the intersections of $M_{1}$ and the elements of $G_{2}$ give a partitioning $G_{1}$ of $M_{1}$.

\section{Questions}

We have already raised certain questions in the preceding sections but we mention some more here.

1. If $M$ is locally simply connected, can it be partitioned into simply connected pieces?

2. If $M$ is a chain from $H$ to $K$ where each of the sets $H$ and $K$ contains only one point, is there an open transformation satisfying the conditions of Theorem 8 ? Whyburn shows such a transformation in [23] if $M$ is 1-dimensional. If the dimension of $M$ is finite, is there such a transformation such that $T^{-1}(x)$ is of dimension less than the dimension of $M$ ?

3. Is there a decreasing sequence of partitionings $G_{1}, G_{2}, \ldots$ of $M$ and a boundary operation carrying subsets of $G_{i}$ into subsets of $G_{i+1}$ that gives a homology theory?

4. Suppose $M_{1}$ and $M_{2}$ are two continuous curves such that one can be given a convex metric with unique segments but the other cannot. Is there an essential difference between the types of partitionings that $M_{1}$ and $M_{2}$ can have?

Many other things could be said concerning partitioning, but there is much to learn. We summarize the results of this paper in one sentence: Any continuous curve can be partitioned in somewhat the same way that a square plus its interior can be partitioned.

\section{REFERENCES}

1. Gustav Beer, Beweis des Satzes, dass jede im kleinen zusammenhängende Kurve convex metrisiert werden kann, Fund. Math. vol. 31 (1938) pp. 281-320.

2. R. H. Bing, A characterization of 3-space by partitianings, Trans. Amer. Math. Soc. vol. 70 (1951) pp. 15-17.

3. - A convex metric for a locally connected continuum, Bull. Amer. Math. Soc. vol. 55 (1949) pp. 812-819.

4. - Complementary domains of continuous curves, Fund. Math. vol. 36 (1949) pp. 306-318.

5. - Higher dimensional hereditarily indecomposable continua, Trans. Amer. Math. Soc. vol. 71 (1951) pp. 267-273. 
6. - Partitioning a set, Bull. Amer. Math. Soc. vol. 55 (1949) pp. 1101-1110.

7. - The Kline sphere characterization problem, Bull. Amer. Math. Soc. vol. 52 (1946) pp. 644-653.

8. R. H. Bing and E. E. Floyd, Coverings with connected intersections, Trans. Amer. Math. Soc. vol. 69 (1950) pp. 387-391.

9. L. M. Blumenthal, Distance geometries, University of Missouri Studies, vol. 13, No. 2, 1938.

10. O. G. Harrold, Jr., Concerning the convexification of continuous curves, Amer. J. Math. vol. 61 (1930) pp. 210-216.

11. W. Hurewicz and H. Wallman, Dimension theory, Princeton University Press, 1948.

12. C. Kuratowski and G. T. Whyburn, Sur les éléments cycliques et leurs applications, Fund. Math. vol. 16 (1930) pp. 305-331.

13. S. Lefschetz, Algebraic topology, Amer. Math. Soc. Colloquium Publications, vol. 27, New York, 1942.

14. K. Menger, Untersuchungen iiber allgemeine Metrik, Math. Ann. vol. 100 (1928) pp. 75-163.

15. E. E. Moise, Grille decomposition and convexification theorems for compact metric locally connected continua, Bull. Amer. Math. Soc. vol. 55 (1949) pp. 1111-1121.

16. - A note of correction, Proceedings of the American Mathematical Society vol. 2 (1951) p. 838.

17. R. L. Moore, Concerning connectedness im kleinen and a related property, Fund. Math. vol. 3 (1922) pp. 232-237.

18. —-, Foundations of point set theory, Amer. Math. Soc. Colloquium Publications, vol. 13, New York, 1932.

19. W. Sierpinski, Sur une condition pour qu'un continu soit une courbe jordanienne, Fund. Math. vol. 1 (1920) pp. 44-60.

20. Garth Thomas, Simultaneous partitionings, Bull. Amer. Math. Soc. Abstracts 57-4-366 and 57-6-553.

21. G. T. Whyburn, Analytic topology, Amer. Math. Soc. Colloquium Publications, vol. 28, New York, 1942.

22. - Concerning S-regions in locally connected continua, Fund. Math. vol. 20 (1933) pp. 131-139.

23. - The existence of certain transformations, Duke Math. J. vol. 5 (1939) pp. 647-655.

24. R. L. Wilder, Topology of manifolds, Amer. Math. Soc. Colloquium Publications, vol. 32, New York, 1949.

25. L. Zippin, On continuous curves and the Jordan curve theorem, Amer. J. Math. vol. 52 (1930) pp. 331-350.

The University of Wisconsin 